\title{
Diversidade de Trichocomaceae Isolada de Solo e Serrapilheira de Floresta Atlântica
}

\author{
Marcelo Elias Fraga ${ }^{1}$, Marcos Gervasio Pereira ${ }^{2}$ \\ ${ }^{1}$ Núcleo de Pesquisa Micológica e Micotoxicológica, Departamento de Microbiologia e Imunologia Veterinária, \\ Universidade Federal Rural do Rio de Janeiro - UFRRJ, Seropédica/RJ, Brasil \\ ${ }^{2}$ Departamento de Solos, Universidade Federal Rural do Rio de Janeiro - UFRRJ, Seropédica/RJ, Brasil
}

\begin{abstract}
RESUMO
O presente trabalho teve como objetivo avaliar a diversidade e a sucessão da família Trichocomaceae em áreas com diferentes graus de antropização, denominadas área mais impactada e área menos impactada. O estudo foi realizado no Parque Natural Municipal do Curió, Paracambi-RJ. Foram selecionadas duas áreas, com diferentes graus de alteração antrópica, sendo em cada uma destas delimitado um talhão de aproximadamente $1.000 \mathrm{~m}^{2}$, sendo nestes realizadas coletas de terra e serrapilheira para a avaliação da fertilidade e da micobiota. De maneira geral, observa-se que, na área mais impactada, ocorreram os maiores valores de $\mathrm{pH}$, Ca e K. Os maiores valores de serrapilheira aportada, observados nesta área, também contribuem para os maiores valores de $\mathrm{K}$, elemento facilmente lixiviável no material em decomposição. Os maiores valores de Unidades Formadoras de Colônias (UFC) no solo e na serrapilheira, $9 \times 10^{4}$ e 5,4 $\times 10^{4}$, respectivamente, foram observados na área mais impactada. Também nesta área foram verificados os maiores valores de Ca no solo e na serrapilheira, o que sugere que esse nutriente pode estar contribuindo para o maior número de UFCs. Para a área menos impactada, os valores de UFC estiveram entre $4 \times 10^{4}$ para serrapilheira e $5 \times 10^{4}$ para o solo, não sendo observada nenhuma associação clara entre um maior número de UFCs e os teores de nutrientes no solo e/ou na serrapilheira. O número total de fungos isolados foi de 87, pertencentes a quatro gêneros e 22 espécies diferentes, sendo 16 Penicillium spp., quatro Aspergillus spp., um Paecilomyices sp. e um Trichoderma sp. Não foi observada uma clara associação entre as variáveis climáticas e o número de UFCs. O maior número de UFCs foi observado na área mais impactada tanto no solo quanto na serrapilheira. Os maiores teores de Ca quantificados nesta área sugerem que esse nutriente pode estar contribuindo para o aumento das UFCs. O genêro Penicillum, em ambas as áreas, foi o mais representativo.
\end{abstract}

Palavras-chave: micobiota, diversidade, impacto do uso do solo.

\section{Diversity of Soil and Litter Isolated Trichocomaceae in Atlantic Forest}

\begin{abstract}
The purpose of the present study was to assess the diversity and succession of family Trichocomaceae in areas with different levels of human disturbance, namely, most impacted area and least impacted area. This research was carried out in "Parque Natural Municipal Curió" (Natural City Preserve) in Paracambi, State of Rio de Janeiro. Two areas with different levels of human disturbance were selected and, in each of these areas, a $1,000 \mathrm{~m}^{2}$ plot was delimited, where soil and leaf litter samplings were collected for fertility and mycobiota assessment. In general, the most impacted area presented the highest values for $\mathrm{pH}, \mathrm{Ca}$ and $\mathrm{K}$. The highest values of leaf litter yield were also observed in this area, which contributed to the higher values of $\mathrm{K}$ - an element easily leachable in mulch. The highest values of Colony Formation Units
\end{abstract}


(CFU) in soil and litter: $5.4 \times 10^{4}$ and $9 \times 10^{4}$, respectively, were observed in the most impacted area. This area also presented the highest values for $\mathrm{Ca}$ in soil and litter, which suggests that this nutrient may contribute to the greater number of CFUs. For the least impacted area, the CFU values were between $4 \times 10^{4}$ for litter and $5 \times 10^{4}$ for soil; no clear association was observed between the higher number of CFUs and the concentrations of nutrients in soil and/or litter. The total number of fungi isolated was 87 , belonging to four different genera and 22 species, with 16 Penicillium spp., four Aspergillus spp., one Paecilomyces sp. and one Trichoderma sp. No clear association was noticed between climatic variables and the number of CFUs. The greatest amount of CFUs was observed in the most impacted area, both in soil and leaf litter. The higher concentration of $\mathrm{Ca}$ quantified in this area suggests that this nutrient may be contributing to the increase in CFUs. The genera Penicillium spp. was the most representative fungus in both areas.

Keywords: mycobiota, diversity, impact of soil use.

\section{INTRODUÇÃO}

O conhecimento acerca das florestas tropicais desempenha papel fundamental na elaboração de estratégias mundiais para a conservação da biodiversidade (Lima \& Guedes-Bruni, 1997). Nesses ambientes, vários estudos têm sido realizados no tocante à avaliação da diversidade florística (Andreata et al., 1997; Tonhasca Junior, 2005), ao aporte e à decomposição de serrapilheira (Pereira et al., 2010; Paula et al., 2009) e à diversidade de microfungos em solo (Abreu \& Pfenning, 2008), porém poucos são os estudos relacionados à família Trichocomaceae.

O Parque Natural Municipal Curió de Paracambi, criado por meio do Decreto Municipal no 1001 de 29 de Janeiro de 2002, foi definido como Unidade de Proteção Integral, com área total de 1.100 ha, e está situado no município de Paracambi-RJ; esse parque está inserido no domínio da Mata Atlântica, formação de Floresta Ombrófila Densa Submontana. Essa Unidade de Conservação (UC) faz parte do corredor de Biodiversidade Tinguá-Bocaina que, por sua vez, está inserido no Corredor da Serra do Mar, tendo como limites a Reserva Biológica do Tinguá e o Parque Nacional da Serra da Bocaina.

Para Peixoto (1991-1992), comparando-se a biodiversidade da Mata Atlântica com outras regiões tropicais, dentre os dados de maior destaque há o grande número de espécies de Myrtaceae e a quantidade de fungos associados à rizosfera. Hawksworth \& Rossman (1997) indicaram que um número alto de espécies de fungos não conhecidas deve ser encontrado em florestas tropicais, no futuro, enfatizando a necessidade de amostragens nessas regiões.

A estimativa mais aceita do número total de espécies de fungos no planeta é de 1,5 milhão, considerada conservadora, pois nesta não foi computada a diversidade de fungos associados a insetos nem a diversidade de fungos endófitos. A proporção de nomes de espécies de fungos descritas versus espécies válidas, apresenta-se com base na proporção de sinonímia de 2,5:1, aumentando em aproximadamente 25 mil o número estimado de espécies válidas. Existem aproximadamente $100 \mathrm{mil}$ espécies de fungos conhecidas, cerca de $7 \%$ do total de 1,5 milhão (Hawksworth, 2001).

$\mathrm{Na}$ biosfera, o habitat mais rico em fungos é o solo. A principal função desses organismos é a decomposição da matéria orgânica, envolvendo pelo menos quatro grupos distintos: celulolíticos, hemicelulolíticos, pectinolíticos e ligninolíticos (Tauk, 1990).

Fungos decompositores têm uma maior importância no ciclo de carbono e nutrientes do ecossistema, porém são sensíveis a distúrbios, poluição e mudanças ambientais (Frankland et al., 1996). O impacto nas mudanças ambientais pode influenciar na diversidade de fungos via decomposição; por isso, é importante conhecer a taxa e o número de decomposição, a frequência de ocorrência e a função das espécies de fungo presentes (Frankland, 1966). 
O baixo nível de diversidade pode corresponder à dominância de poucas espécies, as quais podem apresentar uma vantagem seletiva sobre as demais. As espécies da família Trichocomaceae se enquadram nessas condições; no inverno, quando há baixo índice de chuva, a diversidade aumenta. $\mathrm{O}$ gênero Aspergillus, como um dos principais da família Trichocomaceae, é predominante em solos tropicais e subtropicais (Persiani et al., 1998).

As condições abióticas no interior da camada de serrapilheira, com destaque para a temperatura e a umidade, bem como a composição química da serrapilheira, podem apresentar uma grande variabilidade. Outro fator a ser considerado é a decomposição do material depositado, promovida pelos fungos. Estes possuem capacidades de decomposição distintas em função da produção de enzimas. Tal fato parece uma provável explicação para os efeitos positivos da riqueza de espécies fúngicas na decomposição de serrapilheira (Domsch et al., 1980).

Segundo Attenschwiler et al. (2005), a diversidade de espécies que compõem a serrapilheira e as espécies decompositoras podem influenciar significativamente a decomposição e a liberação de nutrientes para o solo, sendo estes de grande relevância para a comunidade de organismos presentes, bem como para o funcionamento dos ecossistemas. Embora existam poucos estudos acerca do efeito da serrapilheira - em especial, de sua composição química -, na comunidade microbiana do solo (abundância e diversidade), acredita-se que estes atributos possam atuar efetivamente nos organismos do solo.

A partir do exposto, o presente trabalho teve como objetivo avaliar a diversidade e a sucessão da família Trichocomaceae em áreas com diferentes graus de antropização, denominadas área mais impactada e área menos impactada, em um fragmento de Mata Atlântica, localizado em Paracambi-RJ.

\section{MATERIAL E MÉTODOS}

\section{1. Área de estudo}

O Parque Natural Municipal do Curió (coordenadas geográficas: 7.505.350-7,499,750 N e 635.650-632.500 E) possui uma área de aproximadamente 1.100 ha. A área que compreende o Parque pertenceu à extinta Companhia Têxtil Brasil Industrial, que funcionou até o ano de 1996, tendo sido fundada no ano de 1871, após a desativação da Fazenda Ribeirão dos Macacos, onde, em função dos pés de café que são observados, supõe-se que esta era a principal atividade agrícola da área (Figura 1).

A área localiza-se na formação geomorfológica serras e morros altos, na suíte da Serra das Araras, estando localizada na Serra de Paracambi. Para o estudo, foram selecionadas duas áreas, denominadas área mais impactada, definida com alto grau de antropização, e área menos impactada, que apresentava baixo grau de antropização. Os critérios utilizados para a definição das áreas foram os seguintes: a) área mais impactada, localizada próxima a trilhas e onde foi observada a ocorrência de espécies exóticas; b) área menos impactada, que apresentava um maior sub-bosque e maior acúmulo de serrapilheira na superfície do solo. As áreas localizavam-se nas seguintes coordenadas: $22^{\circ} 35^{\prime} 45.1^{\prime \prime}$ S e $43^{\circ} 42^{\prime} 15.1^{\prime \prime}$ WO; $22^{\circ} 35^{\prime} 46.5^{\prime \prime}$ S e $43^{\circ}$ $42^{\prime}$ 17.1" WO, a área com maior grau de antropização, e $22^{\circ} 34^{\prime} 28.3^{\prime \prime}$ S e $43^{\circ} 41^{\prime}$ 09.6” WO; $22^{\circ} 34^{\prime} 26.6^{\prime \prime} \mathrm{S}$ e $43^{\circ} 41^{\prime} 10.6^{\prime \prime}$ WO, a área menos impactada. Em cada uma das áreas, foi delimitado um talhão de aproximadamente $1.000 \mathrm{~m}^{2}$.

Os dados metereológicos utilizados foram temperatura ( $\mathrm{T}$ ) do ar, umidade relativa (UR) e precipitação, sendo estes obtidos da Empresa de Pesquisa Agropecuária do Estado do Rio de Janeiro (Pesagro), na Estação Experimental de Avelar-RJ (Lat.: $22^{\circ} 21^{\prime}$ S e Long.: $43^{\circ} 25^{\prime} \mathrm{W}$ ).

\subsection{Amostragem solo}

Foram coletadas 32 amostras compostas, cada uma delas constituída a partir de dez amostras simples coletadas aleatoriamente. Em cada ponto de amostragem do solo (camada de 5-10 cm), também foram coletadas amostras de serrapilheira. Foram também medidas, no momento das coletas, as temperaturas do solo e da serrapilheira, com auxílio de um geotermômetro. Após a coleta, as embalagens com as amostras foram armazenadas a $4{ }^{\circ} \mathrm{C}$, até o momento da análise. As coletas foram realizadas nos meses de abril, julho, novembro e fevereiro. 


\subsection{Caracterização da micobiota e isolamento}

A micobiota foi quantificada por unidade formadora de colônia (UFC). Os isolamentos foram feitos em $5 \mathrm{~g}$ de solo ressuspendido em $45 \mathrm{~mL}$ de $0,1 \%$ de peptona, contendo $0,1 \%$ de Tween $80 \mathrm{em}$ frasco Erlenmeyer; em seguida, foi agitado por $30 \mathrm{~min}$ em homogeneizador de haste vertical a $150 \mathrm{rpm}$. O meio de cultura utilizado foi Dicloran Rosa de Bengal Cloranfenicol (DRBC), realizado com alíquotas de $0,1 \mathrm{~mL}$ das respectivas diluições $\left(10^{2}\right.$ a $\left.10^{6}\right)$, transferidas para os tubos de cultura contendo $0,9 \mathrm{~mL}$ de meio de cultura. Posteriormente, foi retirado $0,1 \mathrm{~mL}$ dessa solução, que foi plaqueado em forma de gotas, com cinco repetições para cada diluição. As placas de Petri foram incubadas em estufas a $25{ }^{\circ} \mathrm{C}$ por 5 dias. A leitura foi baseada em presença ou ausência de UFC sobre cada gota (Fraga et al., 2010a, b).

\subsection{Identificação da micobiota}

O isolamento foi baseado no estudo morfológico (macroscópico e microscópico) em meio Agar Dicloran Rosa de Bengal Cloranfenicol (DRBC) (Markovina et al., 2005). Para as identificações das espécies, foram utilizados meios específicos para cada grupo de fungo, bem como consultadas bibliografias específicas, como: Klich \& Pitt (1988), Pitt (2000), Samson et al. (2000), Klich (2002), Samson \& Frisvad (2004) e Samson \& Varga (2007).

\subsection{Caracterização do solo e da serrapilheira}

A fertilidade do solo e o teor dos nutrientes trocáveis (potencialmente lixiviáveis) da serrapilheira foram avaliados por meio dos seguintes parâmetros: $\mathrm{pH}$ em água (método do potenciômetro), teor de $\mathrm{Al}$ (trocável) e teor de $\mathrm{K}, \mathrm{Ca}$ e $\mathrm{Mg}$ (trocáveis), para profundidades de $0-10 \mathrm{~cm}$ para solo e a camada superficial de serrapilheira. Os métodos utilizados

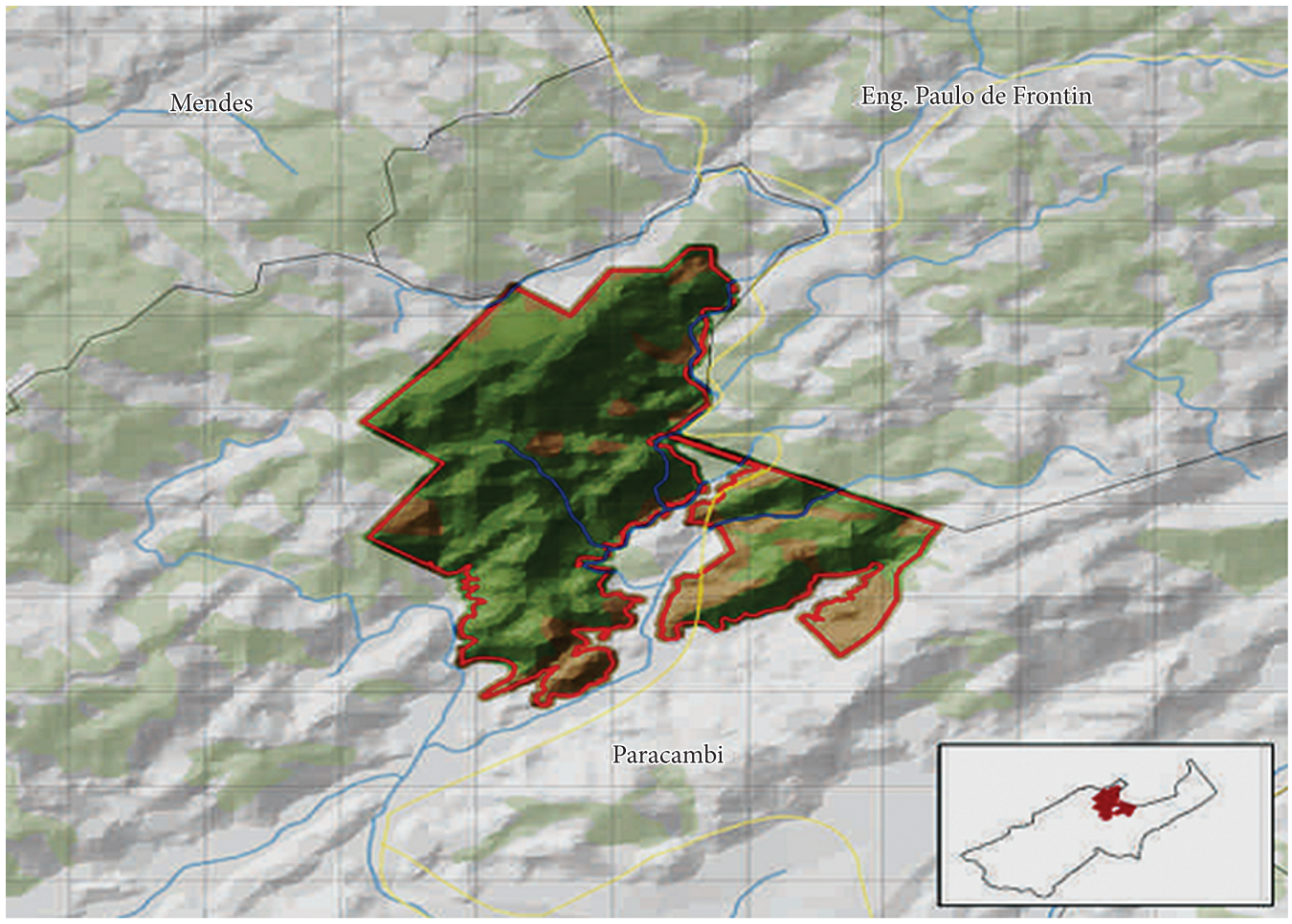

Figura 1. Planta com a localização das áreas de estudo. http://matoecia.blogspot.com.br/2011/11/parque-municipalnatural-do-curio.html Acessado em: 26 de março de 2012.

Figure 1. Plant with the location of sampling areas. http://matoecia.blogspot.com.br/2011/11/parque-municipalnatural-do-curio.html Accessed: March 26, 2012. 
para a determinação do solo e da serrapilheira foram os preconizados pela Embrapa (Embrapa, 1997). Os teores de nutrientes trocáveis da serrapilheira foram determinados de maneira similar aos métodos utilizados para solo. $\mathrm{O}$ delineamento experimental adotado foi inteiramente casualizado e as médias referentes a cada tratamento foram comparadas pelo Teste de Tukey, com nível mínimo de significância de $1 \%$, com o uso do software SISVAR (Universidade Federal de Lavras - UFLA).

\section{RESULTADOS E DISCUSSÃO}

O solo foi classificado como Cambissolo Háplico (Embrapa, 2006), apresentando um baixo grau de desenvolvimento pedogenético. As características topográficas, com destaque para o relevo movimentado, favorecem um elevado grau de remoção de material, por meio do processo erosivo, retardando a evolução do solo.

Os dados ambientais referentes à umidade relativa eà temperatura durante $\mathrm{o}$ ano de coleta apresentaram o valor máximo em novembro para UR, com $82 \%$, e fevereiro para T, com $23{ }^{\circ} \mathrm{C}$, sendo o valor mínimo em julho, com $72 \%$ para UR e $15^{\circ} \mathrm{C}$ para T. Quanto à precipitação, o maior valor observado foi no mês de novembro, com $307,9 \mathrm{~mm}$, e o menor valor, no mês julho, com 1,3 mm. As variações na precipitação, na umidade relativa e na temperatura podem ter promovido um efeito diferenciado entre as áreas, com relação ao desenvolvimento da microbiota (Figura 2).

Na Tabela 1, são apresentados os valores de $\mathrm{pH}, \mathrm{Ca}, \mathrm{K}$ e $\mathrm{P}$, para as áreas mais impactadas e menos impactadas, no solo e na serrapilheira, respectivamente. De maneira geral, observa-se que, na área com maior grau de antropização, ocorreram os maiores valores de $\mathrm{pH}$, Ca e K. Uma possível explicação para os valores mais elevados desses elementos pode ser o fato de estas áreas localizaremse próximas a trilhas utilizadas por animais, os quais podem, pela adição de seus dejetos, ter contribuído para o aumento dos níveis desses nutrientes e do $\mathrm{pH}$, uma vez que nessa área não se observa nenhuma atividade agrícola.

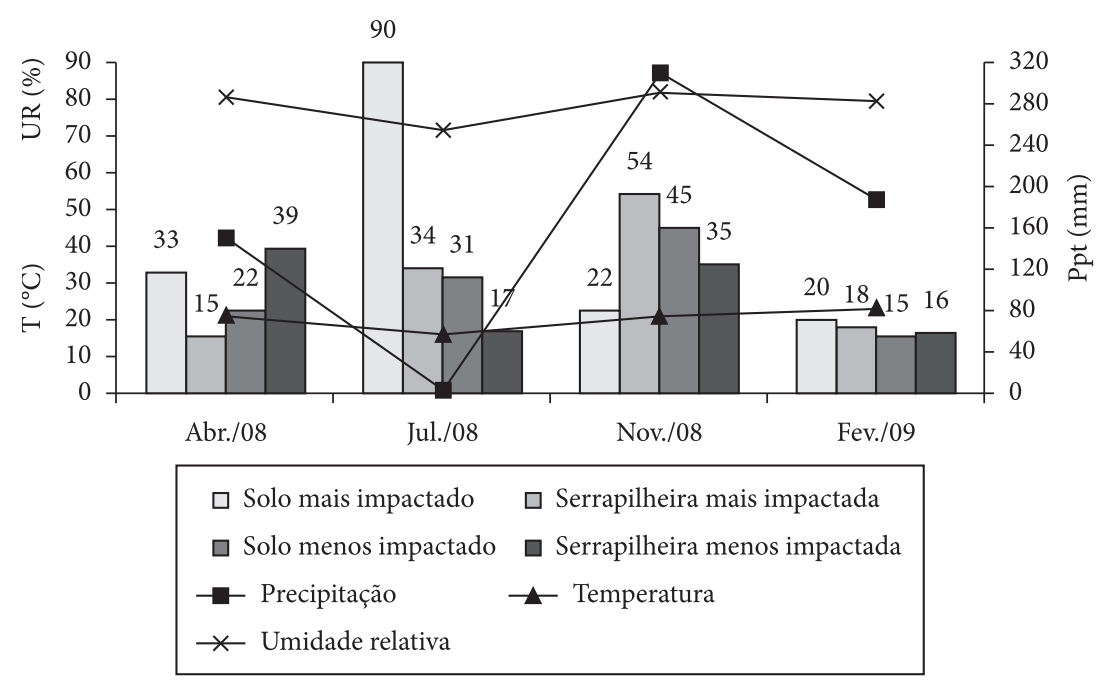

Figura 2. Variação da temperatura $(\mathrm{T})$ em ${ }^{\circ} \mathrm{C}$, umidade relativa (UR) em \%, precipitação (Ppt) em mm (referente à média do mês de coleta) e unidade formadora de colônia $\left(\mathrm{UFC} \times 10^{3}\right)$ de fungo em serrapilheira e solo, nas áreas mais impactadas e menos impactadas (média entre as duas amostras), em relação aos dados metereológicos, no Parque Natural Municipal do Curió, Paracambi-RJ.

Figure 2. Variation of temperature $(\mathrm{T})$ in ${ }^{\circ} \mathrm{C}$, relative humidity $(\mathrm{RH})$ in $\%$, precipitacion $(\mathrm{Ppt})$ in $\mathrm{mm}$ (referring to average for the month of collection) and colony forming unit $\left(\mathrm{CFU} \times 10^{3}\right)$ of fungi in leaf litter and soil in areas impacted and not impacted (average of the two samples) in relation to meteorological data in Parque Natural Municipal do Curió, Paracambi, RJ. 
Para a serrapilheira, de maneira geral, foi verificado um padrão com tendência similar à observada para o solo. Na área com maior grau de antropização, foram quantificados os maiores valores de $\mathrm{pH}$ e Ca. Os maiores valores de serrapilheira aportada, observados nessa área, também contribuem para os maiores valores de $\mathrm{K}$, elemento facilmente lixiviável no material em decomposição.

Para o $\mathrm{pH}$ na área mais impactada, os maiores valores (no solo e na serrapilheira) ocorreram na primeira coleta, com decréscimo nas amostragens seguintes; esse padrão não foi observado na área menos impactada, em que, na serrapilheira, não foi verificada diferença entre as épocas de amostragem e, no solo, o maior e o menor valores de $\mathrm{pH}$ ocorreram na última e na segunda coleta, respectivamente (Tabela 2).

Para o $\mathrm{Ca}$, na área mais impactada, os maiores valores foram verificados na segunda coleta, tanto no solo quanto na serrapilheira, com decréscimo nas coletas seguintes. Esse padrão também foi observado na área menos impactada, no solo e na serrapilheira.

De maneira geral, para o $\mathrm{K}$ e o $\mathrm{P}$, foram observados maiores valores desses nutrientes na primeira coleta, com decréscimo nas seguintes, sendo os menores valores quantificados na última amostragem. Esse padrão ocorreu tanto no solo quanto na serrapilheira, em ambas as áreas estudadas (mais impactada e menos impactada) (Tabela 2).

Os maiores valores de UFC no solo e na serrapilheira, $90 \times 10^{3}$ e $54 \times 10^{3}$, respectivamente, foram observados para a área mais impactada. Também nesta área foram verificados os maiores valores de Ca no solo e na serrapilheira, o que sugere que esse nutriente pode estar contribuindo para o maior número de UFCs. Para a área menos impactada, os valores de UFC estiveram entre

Tabela 1. Médias dos valores de $\mathrm{pH}, \mathrm{Ca}, \mathrm{K}$ e $\mathrm{P}$ no solo e na serrapilheira nas diferentes áreas estudadas no Parque Natural Municipal do Curió, Paracambi-RJ.

Table 1. Values of $\mathrm{pH}, \mathrm{Ca}, \mathrm{K}$ and $\mathrm{P}$ in soil and litter in different areas studied in Parque Natural Municipal do Curió, Paracambi, RJ.

\begin{tabular}{|ccccc|}
\multicolumn{1}{c}{ Área } & \multicolumn{4}{c}{ Solo } \\
& $\mathrm{pH}$ & $\mathrm{Ca}$ & $\mathrm{K}$ & $\mathrm{P}$ \\
\hline Mais impactada & $5,2^{\mathrm{B}}$ & $3,34^{\mathrm{A}}$ & $0,77^{\mathrm{A}}$ & $\mathrm{mg} / \mathrm{kg}$ \\
\hline Menos impactada & $4,7^{\mathrm{A}}$ & $1,31^{\mathrm{A}}$ & $0,61^{\mathrm{B}}$ & $2,75^{\mathrm{A}}$ \\
& \multicolumn{4}{c}{ Serrapilheira } \\
& $\mathrm{pH}$ & $\mathrm{Ca}$ & $\mathrm{K}$ & $\mathrm{P}$ \\
\hline & \multicolumn{4}{c}{$\mathrm{cmol}_{\mathrm{c}} \mathrm{kg}^{-1}$} \\
Mais impactada & $5,3^{\mathrm{A}}$ & $5,53^{\mathrm{A}}$ & $0,55^{\mathrm{B}}$ & $\mathrm{mg} / \mathrm{kg}$ \\
\hline Menos impactada & $5,1^{\mathrm{B}}$ & $3,10^{\mathrm{B}}$ & $1,39^{\mathrm{A}}$ & $15,05^{\mathrm{A}}$ \\
\hline
\end{tabular}

Letras maiúsculas iguais na coluna não diferem pelo teste de Tukey, em 5\%. Média de oito repetições.

Tabela 2. Valores de $\mathrm{pH}, \mathrm{Ca}, \mathrm{Ke} \mathrm{P}$ nas diferentes coletas, nas áreas mais impactada e menos impacatada das amostras de solo e serrapilheira, no Parque Natural Municipal do Curió, Paracambi-RJ.

Table 2. Values of $\mathrm{pH}, \mathrm{Ca}, \mathrm{K}$ and $\mathrm{P}$ in different collections, and most impacted areas less impacted samples of soil and litter in Parque Natural Municipal do Curió, Paracambi, RJ.

\begin{tabular}{|c|c|c|c|c|c|c|c|c|c|c|}
\hline \multicolumn{11}{|c|}{ Solo } \\
\hline $\mathrm{C}$ & \multicolumn{2}{|c|}{ UFC } & \multicolumn{2}{|c|}{$\mathrm{pH}$} & \multicolumn{2}{|c|}{$\mathrm{Ca}$} & \multicolumn{2}{|c|}{ K } & \multicolumn{2}{|c|}{$\mathrm{P}$} \\
\hline & MaI & MeI & $\mathrm{MaI}$ & MeI & $\mathrm{MaI}$ & MeI & $\mathrm{MaI}$ & MeI & MaI & MeI \\
\hline 1 & $33 \times 10^{3}$ & $22 \times 10^{3}$ & $5,73^{\mathrm{a}}$ & $4,58^{\mathrm{BC}}$ & $4,82^{\mathrm{B}}$ & $1,25^{\mathrm{AB}}$ & $2,72^{\mathrm{A}}$ & $2,02^{\mathrm{A}}$ & $5,20^{\mathrm{A}}$ & $5,71^{\mathrm{A}}$ \\
\hline 2 & $90 \times 10^{3}$ & $31 \times 10^{3}$ & $5,24^{\mathrm{B}}$ & $4,36^{\mathrm{C}}$ & $6,55^{\mathrm{A}}$ & $1,70^{\mathrm{A}}$ & $0,28^{\mathrm{B}}$ & $0,33^{\mathrm{B}}$ & $3,89^{\mathrm{A}}$ & $4,66^{A}$ \\
\hline 3 & $22 \times 10^{3}$ & $45 \times 10^{3}$ & $4,96^{\mathrm{BC}}$ & $4,89^{\mathrm{AB}}$ & $1,12^{\mathrm{C}}$ & $1,07^{\mathrm{B}}$ & $0,06^{\mathrm{B}}$ & $0,05^{\mathrm{B}}$ & $0,95^{\mathrm{B}}$ & $0,61^{\mathrm{B}}$ \\
\hline 4 & $20 \times 10^{3}$ & $15 \times 10^{3}$ & $4,88^{\mathrm{C}}$ & $5,09^{\mathrm{A}}$ & $0,85^{\mathrm{C}}$ & $1,35^{\mathrm{AB}}$ & $0,01^{\mathrm{B}}$ & $0,05^{\mathrm{B}}$ & $0,01^{\mathrm{B}}$ & $0,01^{\mathrm{B}}$ \\
\hline \multicolumn{11}{|c|}{ Serrapilheira } \\
\hline $\mathrm{C}$ & \multicolumn{2}{|c|}{ UFC } & \multicolumn{2}{|c|}{$\mathrm{pH}$} & \multicolumn{2}{|c|}{$\mathrm{Ca}$} & \multicolumn{2}{|c|}{ K } & \multicolumn{2}{|c|}{$\mathrm{P}$} \\
\hline & MaI & MeI & MaI & $\mathrm{MeI}$ & MaI & $\mathrm{MeI}$ & $\mathrm{MaI}$ & $\mathrm{MeI}$ & MaI & MeI \\
\hline 1 & $15 \times 10^{3}$ & $39 \times 10^{3}$ & $5,72^{\mathrm{A}}$ & $5,18^{\mathrm{A}}$ & $9,65^{\mathrm{A}}$ & $4,60^{\mathrm{A}}$ & $0,51^{\mathrm{B}}$ & $3,50^{\mathrm{A}}$ & $21,76^{\mathrm{A}}$ & $29,18^{\mathrm{A}}$ \\
\hline 2 & $34 \times 10^{3}$ & $17 \times 10^{3}$ & $5,40^{\mathrm{AB}}$ & $5,23^{\mathrm{A}}$ & $9,90^{\mathrm{A}}$ & $5,75^{\mathrm{A}}$ & $1,66^{\mathrm{A}}$ & $1,99^{\mathrm{B}}$ & $23,43^{\mathrm{A}}$ & $28,27^{\mathrm{A}}$ \\
\hline 3 & $54 \times 10^{3}$ & $35 \times 10^{3}$ & $5,16^{\mathrm{BC}}$ & $5,20^{\mathrm{A}}$ & $1,73^{\mathrm{B}}$ & $1,30^{\mathrm{B}}$ & $0,04^{\mathrm{BC}}$ & $0,08^{\mathrm{C}}$ & $0,57^{\mathrm{B}}$ & $2,41^{\mathrm{B}}$ \\
\hline 4 & $18 \times 10^{3}$ & $16 \times 10^{3}$ & $4,78^{\mathrm{C}}$ & $4,88^{\mathrm{A}}$ & $0,85^{\mathrm{B}}$ & $0,75^{\mathrm{B}}$ & $0,01^{\mathrm{C}}$ & $0,00^{\mathrm{C}}$ & $0,98^{\mathrm{B}}$ & $0,33^{\mathrm{B}}$ \\
\hline
\end{tabular}

Letras maiúsculas na coluna não diferem pelo teste de Tukey, em 5\%. Média de duas repetições. C - Coleta; UFC - Unidade Formadora de Colônia; MaI - Mais impactada; MeI - Menos impactada. 1 - abril/2008; 2 - julho/2008; 3 - novembro/2008; 4 - fevereiro/2009. 
$39 \times 10^{3}$ para serrapilheira e $45 \times 10^{3}$ para o solo, não sendo observada nenhuma associação clara entre um maior número de UFCs e os teores de nutrientes no solo e/ou na serrapilheira (Tabela 2).

Os números de UFCs encontrados apresentamse com valores aproximados aos verificados na literatura. Os valores observados são próximos àqueles verificados por autores em estudos de áreas da Floresta Atlântica, estando esses entre 25 e $38 \times 10^{4}$ UFC. $^{-1}$ de fungos em amostras de solo (Tauk et al., 2005). Vieira \& Nahas (2005) encontraram índices de UFC.g ${ }^{-1}$ de $50 \times 10^{4}$ para fungos em amostras de solo. Em estudos realizados por Bettucci \& Roquebert (1995), em floresta tropical da Malásia, foram observados índices de 2,5 × $10^{4}$ UFC. $g^{-1}$ para solo e $7 \times 10^{4}$ UFC. $^{-1}$ para serrapilheira. Esses valores apresentam-se similares a outros tipos de ambiente, como solo em duna de restinga, com variação de 4,6 × 10 a $9 \times 10^{4} \mathrm{UFC}^{3} \mathrm{~g}^{-1}$ (Fraga et al., 2010b) e ambiente de florestamento, com valores variando de 12,8/58,2 a 20,3/83,3 × $10^{3} \mathrm{UFC}^{-g^{-1}}$ para eucalipto e pinus, respectivamente (Fraga et al., 2010a). Para área de eucalipto, não foi observada redução da micobiota no ambiente impactado. Alguns estudos apontam que as folhas de eucalipto são muito ricas em taninos, fenóis, óleos e outras substâncias que oferecem maior resistência à ação dos fungos no processo de degradação.

O número total de fungos isolados foi de 87, pertencentes a quatro gêneros e 22 espécies diferentes, sendo 16 Penicillium spp., quatro Aspergillus spp., um Paecilomyices sp. e um Trichoderma sp. Entre as 22 espécies isoladas, sete espécies (A.flavus, A. niger, P. aurantiogriseum, P. janczewskii, P. oxalicum, P. rugulosum e Trichoderma) não foram encontradas em solo, três espécies ( $P$. citreonigrum, $P$. crustosum e P. felutanum) não foram encontradas em serrapilheira

Tabela 3. Espécies de Trichocomaceae isoladas do solo e da serrapilheira no período de um ano no Parque Natural Municipal do Curió, Paracambi, RJ.

Table 3. Species of Trichocomaceae isolated from soil and litter in one-year period in Parque Natural Municipal do Curió, Paracambi, RJ.

\begin{tabular}{|c|c|c|c|c|}
\hline \multirow[b]{2}{*}{ Fungos } & \multicolumn{2}{|c|}{ Solo } & \multicolumn{2}{|c|}{ Serrapilheira } \\
\hline & $\begin{array}{l}\text { Área mais } \\
\text { impactada }\end{array}$ & $\begin{array}{l}\text { Área menos } \\
\text { impactada }\end{array}$ & $\begin{array}{l}\text { Área mais } \\
\text { impactada }\end{array}$ & $\begin{array}{c}\text { Área menos } \\
\text { impactada }\end{array}$ \\
\hline Aspergillus sp. & $\mathrm{x}$ & & $\mathrm{x}$ & $\mathrm{x}$ \\
\hline A. flavus & & & & $\mathrm{x}$ \\
\hline A. fumigatus & $\mathrm{x}$ & $\mathrm{x}$ & & $\mathrm{x}$ \\
\hline A. niger & & & $\mathrm{x}$ & \\
\hline Paecilomyces sp. & $\mathrm{x}$ & $\mathrm{x}$ & & $\mathrm{x}$ \\
\hline Penicillium sp. 1 & $\mathrm{x}$ & $\mathrm{x}$ & $\mathrm{x}$ & $\mathrm{x}$ \\
\hline Penicillium sp. 2 & $\mathrm{x}$ & $\mathrm{x}$ & $\mathrm{x}$ & $\mathrm{x}$ \\
\hline P. aurantiogriseum & & & $\mathrm{x}$ & \\
\hline P. brevicompactum & $\mathrm{x}$ & $\mathrm{x}$ & $\mathrm{x}$ & \\
\hline P. citreonigrum & $\mathrm{x}$ & $\mathrm{x}$ & & \\
\hline P. corylophilum & $\mathrm{x}$ & $\mathrm{x}$ & $\mathrm{x}$ & $\mathrm{x}$ \\
\hline P. crustosum & $\mathrm{x}$ & & & \\
\hline P. felutanum & $\mathrm{x}$ & & & \\
\hline P. implicatum & & $\mathrm{x}$ & & $\mathrm{x}$ \\
\hline P. janczewskii & & & & $\mathrm{x}$ \\
\hline P. miczynskii & $\mathrm{x}$ & $\mathrm{x}$ & & $\mathrm{x}$ \\
\hline P. oxalicum & & & $\mathrm{x}$ & \\
\hline P. paxilli & & $\mathrm{x}$ & $\mathrm{x}$ & \\
\hline P. rugulosum & & & $\mathrm{x}$ & $\mathrm{x}$ \\
\hline P. variable & $\mathrm{x}$ & & $\mathrm{x}$ & \\
\hline P. verruculosum & $\mathrm{x}$ & & & $\mathrm{x}$ \\
\hline Trichoderma sp. & & & $\mathrm{x}$ & \\
\hline
\end{tabular}


e as outras 12 espécies foram encontradas em ambos os habitats (Tabela 3).

Espécies de Trichocomaceae têm sido citadas e frequentemente registradas em investigações ecológicas de solos na América do Norte e na Europa. Entretanto, relativamente pouco se conhece acerca da diversidade e da distribução desses fungos em ambientes naturais, com exceção dos solos da América do Norte e da Europa (Markovina et al., 2005). No Brasil, são encontrados poucos estudos direcionados para espécies de Trichocomaceae em ambientes de florestas tropicais. Os relatos são citações de algumas espécies em diferentes estudos, como: 'A Ocorrência de Aspergillus e Penicillium em solo na região norte (Pará e Maranhão)', por Batista et al. (1967a, b), 'Solo de Duna no Rio de Janeiro (Fraga et al., 2010b)' e 'Ambiente de Florestamento de Pinus e Corymbia' (Fraga et al., 2010a).

Espécies de Penicillium e Aspergillus crescem rapidamente e produzem grandes quantidades de conídios, que são facilmente dispersos. Por isso, esses fungos ocorrem em uma ampla variedade de ambientes. De acordo com Christensen et al. (2000), Penicillia pode representar 0 a $67 \%$ (média de 35\%) das espécies prevalentes em qualquer comunidade de fungo no solo sob vegetação nativa, onde a diversidade atinge habitualmente 50 a $75 \%$ - ou mais espécies - por grama de solo. Neste estudo, foram isoladas 16 espécies de Penicillium de um total de 22 espécies isoladas. Os números observados corroboram com os dados de estudos prévios reportados.

\section{CONCLUSÕES}

O maior número de UFCs foi observado na área menos impactada tanto no solo quanto na serrapilheira, o que sugere que este pode ser um indicador do distúrbio ao qual a área foi submetida. Os maiores teores de Ca quantificados na área menos impacatada indicam que este nutriente pode estar contribuindo para o aumento das UFCs. Quanto ao número de isolados, foram quantificados 87 e, dentre estes, 22 espécies se repetiram entre coletas, sendo o genêro Penicillum, em ambas as áreas, o mais representativo, com 16 espécies.

\section{STATUS DA SUBMISSÃO}

Recebido: 07/07/2011

Aceito: 14/06/2012

Publicado: 31/12/2012

AUTOR(ES) PARA CORRESPONDÊNCIA

\section{Marcelo Elias Fraga}

Núcleo de Pesquisa Micológica e

Micotoxicológica,

Departamento de Microbiologia e

Imunologia Veterinária,

Universidade Federal Rural do

Rio de Janeiro - UFRRJ,

Rod. BR 465, Km 7, CEP 23890-000,

Seropédica, RJ, Brasil

e-mail: fraga@ufrrj.br

\section{REFERÊNCIAS}

Abreu LM, Pfenning LH. Diversidade de microfungos em solos tropicais. In: Moreira FMS, Siqueira JO, Brussaard L. Biodiversidade do solo em ecossistemas brasileiros. Lavras: Editora UFLA; 2008. p. 445-481.

Andreata RHP, Gomes M, Baumgratz JFA. Plantas herbáceo-arbustivas terrestres da Reserva Ecológica de Macaé de Cima. In: Lima HC, Guedes-Bruni RR, editors. Serra de Macaé de Cima: Diversidade Florística e Conservação em Mata Atlântica. Rio de Janeiro: Jardim Botânico do Rio de Janeiro; 1997.

Attenschwiler SH, Tiunov AV, Scheu S. Biodiversity and litter and decomposition terrestrial ecosystems. Annual Review of Ecology, Evolution, and Systematics 2005; 36: 191-218. http://dx.doi. org/10.1146/annurev.ecolsys.36.112904.151932

Batista AC, Silvia JO, Maciel MJP, Almeida AG. Aspergillaceae dos solos das zonas fisiográficas de Bragança e do Baixo Amazonas, Estado do Pará. Atas Instituto Micologia 1967a; 4: 185-229.

Batista AC, Silvia JO, Maciel MJP, Lima JA, Moura NR. Micropopulações fúngicas dos solos de Território Federal do Amapá. Atas Instituto Micologia 1967b; 4: 117-121.

Bettucci L, Roquebert L. Studies on microfungi from tropical rain forest litter and soil. Nova Hedwigia 1995; 6: 111-118.

Christensen M, Frisvad JC, Tuthill DE. Penicillium species diversity in soil and some taxonomic and ecological notes. In: Samson R, Pitt J. Integration of Modern Taxonomic Methods for Penicillium and 
Aspergillus Classification. Londom: Harwood Academic Publishers; 2000. p. 309-321.

Domsch KH, Gams W, Anderson T. Compendium of soil fungi. London: Academic Press; 1980. vol. 1, 860 p.

Embrapa. Manual de métodos de análise de solo. Rio de Janeiro: Embrapa Solos; 1997. 212 p.

Embrapa. Centro Nacional de Pesquisa de Solos CNPS. Sistema Brasileiro de Classificação de Solos. 2. ed. Rio de Janeiro: EMBRAPA Solos; 2006. 306 p.

Fraga ME, Pereira MG, Barbosa DJ, Melo MP. Diversidade de Trichocomaceae isoladas de solo em dois ecossistemas florestais. Ciência Florestal 2010a; 20: 169-177.

Fraga ME, Pereira MG, Souza FA. Micobiota do solo de uma área de duna na Restinga da Marambaia, Rio de Janeiro, RJ. Floresta e Ambiente 2010b; 17: 30-36. http:// dx.doi.org/10.4322/floram.2011.007

Frankland JC. Fungal of fungi on decaying braken petioles. Journal of Ecology 1966; 57: 25-36. http:// dx.doi.org/10.2307/2258205

Frankland JC, Magan N, Gadd GM. Fungi and environmental change. Cambridge: Cambridge University Press; 1996. 368 p.

Hawksworth DL. The magnitude of fungal diversity: the 1.5 million species estimate revisited. Mycological Research 2001; 105: 1422-1432. http://dx.doi. org/10.1017/S0953756201004725

Hawksworth DL, Rossman AY. Where are all the undescribed fungi? Phytopathology 1997; 87(9): 888891. PMid:18945058. http://dx.doi.org/10.1094/ PHYTO.1997.87.9.888

Klich MA. Identification of common Aspergillus species. Utrecht: CBS; 2002. 116 p.

Klich MA, Pitt JI. A laboratory guide to common Aspergillus species and their teleomorphs. North Ryde: CSIRO; 1988. $116 \mathrm{p}$.

Lima HC, Guedes-Bruni RR, editors. Serra de Macaé de Cima: Diversidade Florística e Conservação em Mata Atlântica. Rio de Janeiro: Instituto de Pesquisas Jardim Botânico do Rio de Janeiro; 1997. 346 p.

Markovina A, Pitt JI, Hocking AD, Carter DA, McGee PA. Diversity of the Trichocomaceae in the Katandra Nature Reserve, Central Coast, NSW, Australia. Mycological
Research 2005; 109: 964-973. PMid:16209302. http:// dx.doi.org/10.1017/S0953756205003540

Paula RR, Pereira MG, Menezes LFT. Aporte de nutrientes e decomposição da serrapilheira em três fragmentos florestais periodicamente inundados na Ilha da Marambaia, RJ. Ciência Florestal 2009; 19: 139-148.

Peixoto AL. Vegetação da Costa Atlântica. In: Monteiro S, Kaz L, coordenadores. Floresta Atlântica. Rio de Janeiro: Alumbramento; 1991-1992. p. 33-41.

Pereira MG, Loss A, Beutler SJ, Torres JLR. Carbono, matéria orgânica leve e fósforo remanescente em diferentes sistemas de manejo do solo. Pesquisa Agropecuária Brasileira 2010; 45: 508-514. http://dx.doi. org/10.1590/S0100-204X2010000500010

Persiani AM, Maggi O, Casado MA, Pineda FD. Diversity and varialility in soil fungi from a disturbed tropical rain forest. Mycologia 1998; 90: 206-214. http:// dx.doi.org/10.2307/3761296

Samson RA, Hoekstra ES, Frisvad JC, Filtenborg O. Introduction to food and airborne fungi. 6th ed. Baarn: CBS; 2000. 389 p.

Samson RA, Frisvad JC. Penicillium subgenus Penicillium: new taxonomic schemes, mycotoxins and other extrolites. Studies in Mycology 2004; 49: 1-251.

Samson RA, Varga J. Aspergillus systematic in the genomic era. Studies in Mycology 59. Utrecht: CBS Fungal Biodiversity Centre; 2007. 207 p.

Pitt JI. A laboratory guide to common Penicillium species. Australia: Food Science Australia a Joint Venture of CSIRO; AFISC; 2000. 197 p.

Tauk SM. Biodegradação de resíduos orgânicos no solo. Revista Brasileira de Geociências 1990; 20(1-4): 299-301.

Tauk SM, Grrlipp A, Ruegger M, Attili DS, Malagutti E. Soilborne filamentous fungi in Brasil. Journal Basic Microbiological 2005; 45: 72-82. http://dx.doi. org/10.1016/j.micres.2005.01.004

Tonhasca Junior, A. Ecologia e História Natural da Mata Atlântica. Rio de Janeiro: Interciência; 2005. PMid:15678565. http://dx.doi.org/10.1002/ jobm.200410418

Vieira FCS, Nahas E. Comparison of microbiol numbers in soils by using various culture media and temperatures. Microbiological Research 2005; 160: 197-202. 